\title{
Caracterización Viscoelástica de Biopelículas Obtenidas a Base de Mezclas Binarias
}

\author{
Rafael E. González Cuello(1)*, Néstor A. Urbina ${ }^{(2)}$ y Lena Morón Alcázar ${ }^{(1)}$ \\ (1) Universidad de Cartagena, Piedra de Bolívar - Av Del Consulado, Calle 30 № 48-157., Colombia. \\ (e-mail: rgonzalezc1@unicartagena.edu.co) \\ (2) Universidad Francisco de Paula Santander, Departamento de Medio Ambiente. Av Gran Colombia \\ №12E - 96, Barrio Colsag., Colombia.
}

${ }^{*}$ Autor a quien debe ser dirigida la correspondencia

Recibido Oct. 29, 2014; Aceptado Ene. 5, 2015; Versión final recibida Ene. 18, 2015

\begin{abstract}
Resumen
El objetivo de este trabajo fue caracterizar la viscoelasticidad dinámica lineal de biopelículas obtenidas con glicerol al $12 \%(\mathrm{v} / \mathrm{v})$, goma gelana (GG), concentrado de proteína de suero lácteo (CPS) y sus mezclas. Las biopelículas basadas en biopolímeros funcionales y concentrado de proteína de suero lácteo (CPS) son una alternativa en la industria alimentaria al empaque tradicional de alimentos con materiales sintéticos, por lo cual se hace necesario realizar estudios iniciales de tipo técnico. Para la caracterización de las biopelículas se utilizó análisis reológico dinámico y curvas de Creep ajustadas al modelo de Burgers. Todas las biopelículas estudiadas se comportaron como sistemas reológicos con características predominantemente elásticas. El comportamiento de los módulos dinámicos puede ser un indicativo de la formación de redes interdependientes de la GG y el CPS. Por tanto, es posible utilizar estos materiales individualmente 0 en mezclas, para la obtención de biopelículas activas y recubrir diversos sistemas alimentarios sólidos.
\end{abstract}

Palabras clave: goma gelana, proteína de suero lácteo, biopelículas, viscoelasticidad

\section{Viscoelastic Characterization of Biofilms obtained from Binary Mixtures}

\begin{abstract}
The aim of this study was to characterize the lineal dynamic viscoelasticity of biofilm obtained with $12 \%$ glycerol (v/v), gellan gum (GG), whey protein concentrate (WPC) and their mixtures. Biofilms based on functional biopolymer and whey protein concentrate (WPC) are an alternative in the food industry to traditional packaging with synthetic materials, so it is necessary to make preliminary technical studies. Dynamic rheological analysis and Creep curves adjusted to Burgers model were used to characterize the biofilms. The results showed that all biofilms behaved as rheological system with predominantly elastic characteristics. The behavior of dynamic moduli can be a sign of interdependent network formation of GG and WPC. It is therefore possible to use these materials individually or in mixture to obtain active biofilm and coating various solid food systems.
\end{abstract}

Keywords: gellan gum, whey protein, biofilms, viscoelasticity 


\section{INTRODUCCIÓN}

Las películas y los recubrimientos comestibles han sido considerados particularmente para la preservación de alimentos debido a que pueden mejorar la calidad global de algunos alimentos (Franssen y Krochta, 2003). Las biopelículas pueden ser utilizadas para cubrir superficies alimentarias, separar zonas e ingredientes incompatibles, formar una barrera contra el oxígeno, aromas, etc. Entre otras características importantes, también pueden ser empleadas como acarreadores de agentes funcionales tales como antioxidantes y sustancias antimicrobianas para mejorar la seguridad y estabilidad de alimentos (Lin y Zhao, 2007). La producción de biopelículas con materiales poliméricos biodegradables es conveniente debido a las bajas consecuencias ambientales comparadas con los materiales plásticos sintéticos utilizados generalmente para el empacado de alimentos. Diversos materiales han sido utilizados como base para la elaboración de biopelículas, todos reconocidos como seguros para consumo humano. Entre ellos se encuentran polisacáridos, lípidos y proteínas. Los de mayor aplicación son las proteínas de suero, caseína, proteína de soja y algunas gomas.

En cuanto a las proteínas de suero lácteo, estas representan, el $20 \%$ del total de las proteínas en la leche; comercialmente conocidos como concentrados de proteína de suero lácteo (CPS) por su contenido en proteínas entre 25 - $80 \%$. Las principales proteínas de suero, la $\beta$-lactoglobulina y la $\alpha$-lactoalbumina al ser desnaturalizadas por el calor producen películas transparentes e insípidas. La goma gelana, es un heteropolisacarido lineal aniónico producido por la bacteria Sphingomonas paucimobilis y consiste de unidades de repetición de un tetrasacárido (1,3- $\beta$-D-Glucosa; 1,4- $\beta$-D-Acido glucuronico; $1,4 \beta$-D Glucosa; y 1,4 $\alpha$-L-ramnosa), (González et al. 2012).

La caracterización reológica es importante en la formulación, procesamiento, transporte y almacenamiento de alimentos, además es una herramienta útil en el diseño y predicción de la estabilidad de muestras almacenadas (Acevedo et al. 2014a). Además, proporciona información sobre los mecanismos de gelificación y ayudan a mejorar los atributos texturales impartidos por los biopolímeros y las proteínas a las biopelículas. Las curvas de creep/recuperación son obtenidas en términos de compliancia $\mathrm{J}(\mathrm{t})$, que es la relación cuantitativa entre el esfuerzo aplicado a la muestra y la deformación resultante. La representación matemática de la relación esfuerzo/recuperación utiliza diferentes modelos incluyendo resortes y amortiguadores. Cuando estos elementos son alineados en serie o en paralelo, determinan las propiedades viscoelásticas de materiales sólidos y fluidos. Existen ecuaciones matemáticas que relacionan el esfuerzo aplicado con la deformación resultante (Steffe, 1996). Los principales modelos que describen el comportamiento viscoelástico de un sistema son: Maxwell, Kelvin-Voigt y Burgers. En el presente estudio se utilizó el modelo de Burgers que proporciona una aproximación real al comportamiento viscoelástico de numerosos geles y alimentos (González, 2011).

La viscoelasticidad significa la existencia simultanea de propiedades viscosas y elásticas en el mismo material, por tanto, es razonable asumir que todos los materiales tienen una naturaleza viscoelástica; sin embargo, es importante mencionar que lo anterior es válido bajo ciertas circunstancias (Steffe, 1996). Por muchos años, los trabajos científicos han estado enfocados en determinar la viscoelasticidad lineal de los materiales, esto es debido principalmente a tres razones: se puede dilucidar la estructura molecular de los materiales, los parámetros y funciones medidos pueden ser útil en el control de calidad de ciertos productos industriales y finalmente porque el conocimiento de la viscoelasticidad lineal es muy útil en diversas áreas de la industria (Malkin, 1994).

En las biopelículas se han evaluado la capacidad biodegradable (González y Álvarez, 2013), antioxidante (Hua Li et al. 2014), la viscoelasticidad por espectroscopia de biopelículas microbianas (Lau et al. 2009), la viscoelasticidad de películas de almidón de tapioca en función de la concentración del glicerol (Chen et al. 2009), la reología de películas a base de gelana de alto y bajo acilo conteniendo ácido ascórbico (León et al. 2009), de las dispersiones formadoras de biopelículas (Peressini et al. 2003), de películas comestibles a base de colágeno (Deiber et al. 2011) pero pocos estudios se han enfocado en la viscoelasticidad lineal de biopelículas a base de CPS y GG. Por tanto, el objetivo del siguiente trabajo fue caracterizar el comportamiento viscoelástico de biopelículas elaboradas a base de goma gelana y proteína de suero lácteo utilizando $\mathrm{CaCO}_{3}$ como agente entrecruzante, ácido cítrico como agente inductor del gel y glicerol como plastificante.

\section{MATERIALES Y MÉTODOS}

\section{Materiales}

La goma gelana fue suministrada por modernist pantry (USA). La proteína de suero lácteo por ingredientes y productos funcionales (IPF-Colombia), el glicerol ( $87 \%$ de pureza) y el Carbonato de Calcio por Merck mientras que el ácido cítrico fue suministrado por Pancreac (USA). 


\section{Preparación de las biopelículas}

Para la elaboración de las biopelículas se utilizó un diseño de mezclas simples así: GG, CPS y sus mezclas 25GG/75CPS, 50GG/50CPS, 75GG/25CPS disueltas en agua destilada conteniendo $30 \mathrm{mM}$ de $\mathrm{CaCO}_{3}$. Posteriormente se adicionó glicerol como plastificante en una proporción al $12 \%(\mathrm{v} / \mathrm{v})$ y se ajustó el pH. Finalmente, las preparaciones fueron sometidas a $90{ }^{\circ} \mathrm{C}$ por 30 minutos en baño termostatado. Para la formación de las biopelículas fueron vertidos volúmenes de $15 \mathrm{~mL}$ de las soluciones anteriores en cajas de Petri. El secado se realizó en estufa a $30^{\circ} \mathrm{C}$ durante 45 horas aproximadamente asegurando uniformidad de las biopelículas.

\section{Estudio reológico dinámico}

Las determinaciones fueron llevadas a cabo en un reómetro de bajo esfuerzo (Paar Physica). Las muestras fueron colocadas en el plato inferior (Peltier) y el Gap fue ajustado. Las determinaciones se llevaron a cabo en un rango de velocidades de cizalla comprendidas entre $5.00 \times 10^{-5}$ y $1.00 \times 10^{3} \mathrm{~s}^{-1}$ y esfuerzos de cizalla entre $6.37 \times 10^{-1}$ y $6.37 \times 10^{3} \mathrm{~Pa}$ utilizando una geometría PP20.

\section{Viscoelasticidad lineal}

Se realizó un barrido de amplitud con torques entre $1 \times 10^{-3}$ y $1 \times 10^{1} \mathrm{mNm}$ para determinar la zona viscoelástica lineal, posteriormente se llevó a cabo un barrido de frecuencia entre 0,010 y $1,50 \mathrm{~Hz}$, para determinar el comportamiento de los módulos de almacenamiento (G') y de pérdida (G') en función de la frecuencia angular.

\section{Curvas de Creep-recuperación}

Esta determinación consiste en la aplicación de un esfuerzo y su subsecuente suspensión para evaluar la recuperación del sistema y los parámetros característicos que representan este comportamiento. Los resultados están en términos de compliancia ajustados al modelo de Burgers (ecuación 1).

$J(t)=J_{0}+J_{1}\left(1-e^{t / \lambda r e t}\right)+\eta / t$

Donde $J_{0}$ corresponde a la compliancia instantánea, $\eta$ a la viscosidad newtoniana. $J_{1}$ a la compliancia retardada, $\lambda_{\text {ret }}$ es el tiempo de retardo de la unidad de Kelvin y t finalmente representa el tiempo de análisis.

\section{Método de análisis de datos}

Los datos fueron analizados mediante análisis normal de varianza (ANOVA un factor) utilizando la prueba de tukey con el fin de establecer diferencias estadísticas significativas $(p<0.05)$ entre las muestras analizadas. Para tal fin se utilizó el programa de computo SPSS (ver 17.0 para Windows).

\section{RESULTADOS Y DISCUSIÓN}

En la Figura 1 se muestra el comportamiento de G' y G" con respecto a la frecuencia para las biopelículas analizadas. Se puede apreciar la existencia de diferencias estadísticas significativas $(P<0,05)$ en el espectro para las magnitudes de los parámetros viscoelásticos de las biopelículas, igualmente se observa un mayor comportamiento elástico que viscoso (G'>G') en los intervalos de frecuencia estudiados $(0,01-1,50 \mathrm{~Hz})$ y una mínima dependencia de estos módulos con la frecuencia, ya que es un comportamiento característico de un gel fuerte de acuerdo a la clasificación de Clark y Ross-Murphy (1987). Los mayores valores de G' se encontraron en las biopelículas elaboradas con CPS, lo que significa que estas biopelículas tienen una estructura que requiere de mayores esfuerzos de cizalla para su deformación, este comportamiento ha sido observado en diferentes sistemas alimenticios como queso tipo Manchego (González, 2011) yogurt con microcápsulas (González et al. 2014) y sueros costeños (Acevedo et al. 2014b). Es interesante mencionar que los menores valores para G' fueron obtenidos para las biopelículas elaboradas a base de GG como una consecuencia de las características hidrofílicas de este biopolímero al formar redes llenas de agua en presencia de cationes divalentes como el $\mathrm{Ca}++$. Mientras que las biopelículas obtenidas con las mezclas (CPS/GG) presentaron valores intermedios en sus respectivos módulos. Por lo mencionado anteriormente, es posible que exista la formación de redes interdependientes entre los dos compuestos, es decir que no exista una interacción entre la GG y el CPS.

Los valores de tan $\delta$ para las biopelículas estudiadas están representados en la figura 2 donde se puede apreciar que el incremento en las concentraciones de CPS disminuye significativamente $(P<0,05)$ los valores de $\tan \delta$ en todo el intervalo de frecuencia estudiado, lo cual indica que en este tipo de biopelículas predominan más las propiedades elásticas que en las biopelículas con mayor concentración de goma gelana, este comportamiento probablemente obedece a la formación de estructuras más complejas, lo cual 
acentúa el carácter viscoelástico (Lucey et al. 1998). Comportamientos opuestos han sido reportados por otros autores (Chillo et al. 2008) quienes obtuvieron un leve aumento en los valores de tan $\delta$ cuando incrementaban la concentración de quitosano y glicerol en la formación de películas esta diferencia probablemente se debe al efecto del glicerol sobre el movimiento de las cadenas al actuar como plastificante (Liang et al. 2014).

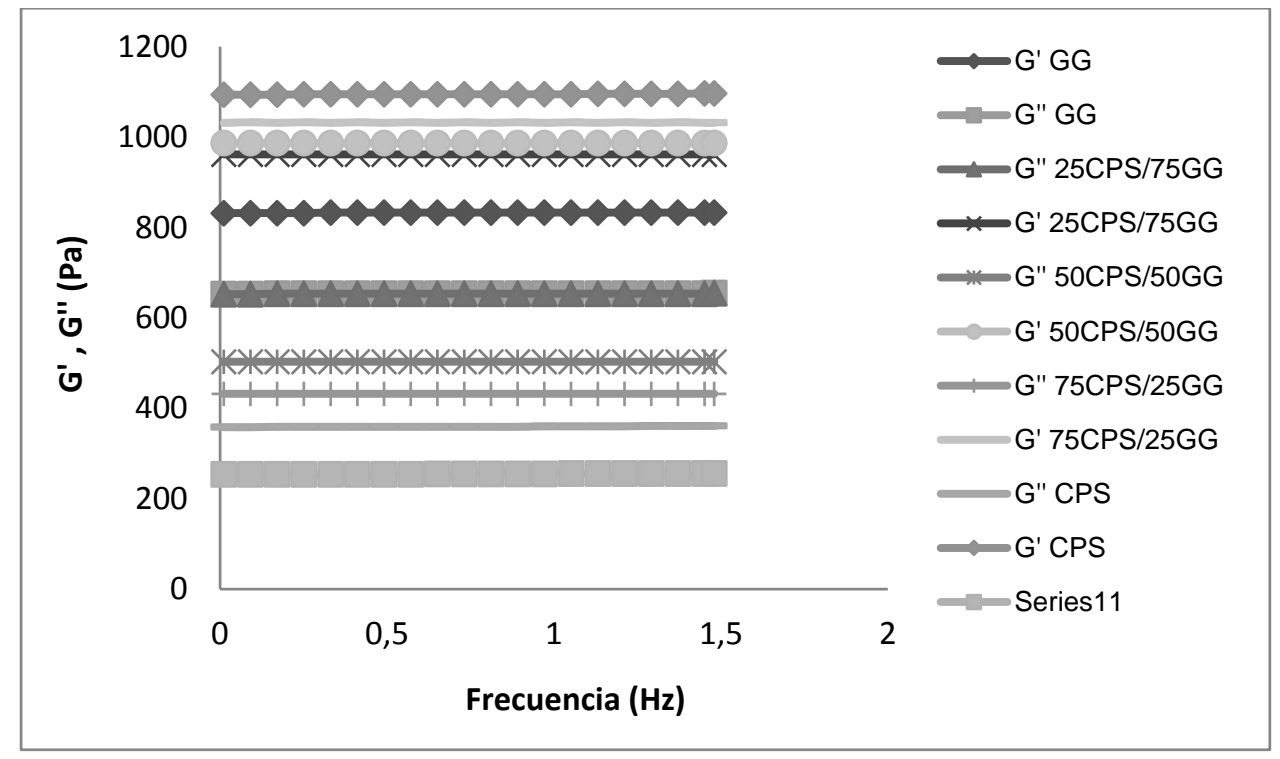

Fig. 1: Comportamiento de los módulos dinámicos G' y G" en función de la frecuencia de biopelículas de GG, CPS y sus mezclas

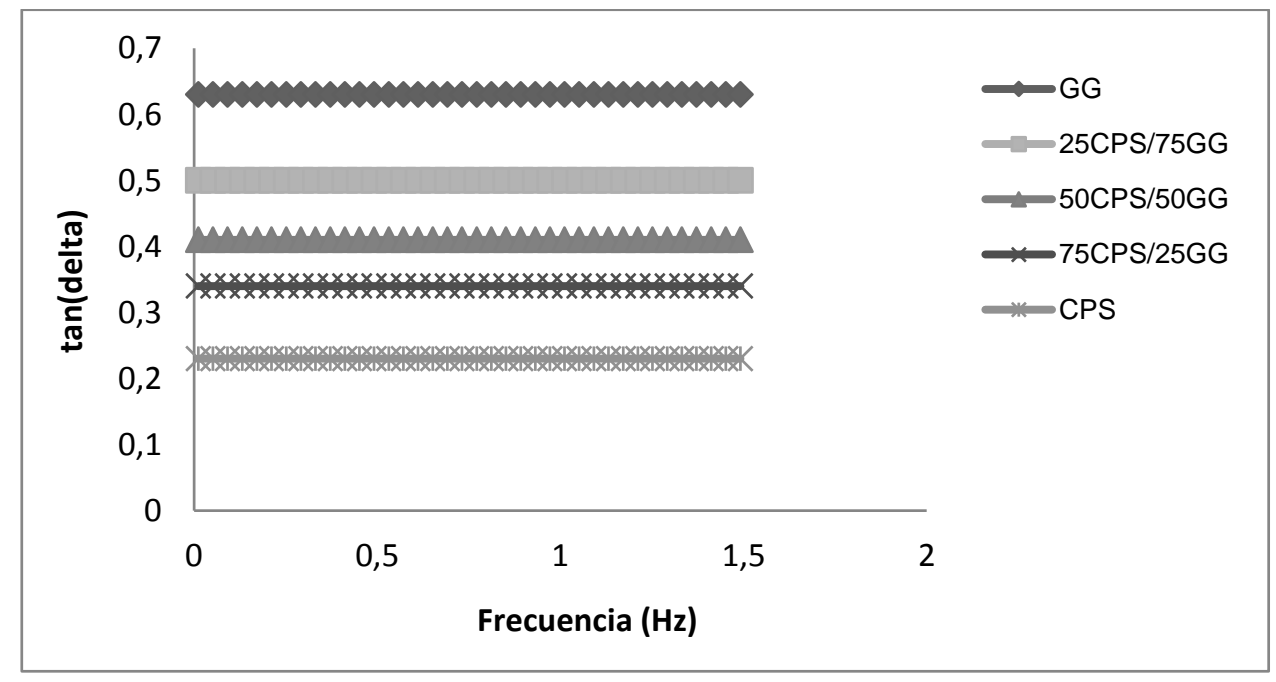

Fig.2: valores de la tangente del ángulo de fase $(\delta)$ en función de la frecuencia para las biopelículas estudiadas

En todos los análisis el comportamiento de las biopelículas fue similar a los de un sólido viscoelástico. Sin embargo, la concentración de CPS contribuye en mayor medida con el carácter elástico de las biopelículas. La elasticidad es el comportamiento reversible de esfuerzo/deformación, el cual es medido como el reciproco de $\delta$, donde los sólidos puramente elásticos tienen el ángulo de fase de $0^{\circ} \mathrm{y}$ el fluido viscoso tiene un ángulo de fase de $90^{\circ}$ (Silva et al. 2013).

\section{Curvas de Creep}

Las curvas de Creep fueron modeladas matemáticamente utilizando la ecuación de Burgers obteniendo sus respectivos parámetros. En todas las biopelículas estudiadas se presentó recuperación cuando el esfuerzo aplicado fue suspendido, por lo tanto, se comportaron como sólidos viscoelásticos, concordando con los resultados obtenidos en los módulos de almacenamiento y pérdida. La menor recuperación se observó en las biopelículas elaboradas con GG como consecuencia de la interacción entre las cadenas laterales de la goma gelana (González et al. 2012) mientras que la mayor recuperación se presentó en las biopelículas 
elaboradas con CPS. En términos generales, las biopelículas mostraron recuperaciones menores que los geles elaborados con biopolímeros funcionales (goma gelana y almidón de maíz), (González et al. 2012. Jiménez et al. 2005). En la Tabla 1 se muestran los parámetros reológicos del modelo de Burgers para las biopelículas de GG, CPS y sus mezclas GG/CPS, así como los valores de recuperación, para cada biopelícula, obtenidas en la región viscoelástica lineal $(R V L),(0,0045$ y 0,0052 mNm). Las biopelículas elaboradas a base de CPS presentaron la mayor viscosidad $\left(4,67 \times 10^{6}\right.$ Pas), por el contrario aquellas biopelículas elaboradas a base de GG mostraron la menor viscosidad $\left(8,23 \times 10^{4} \mathrm{Pas}\right)$ y sus mezclas presentaron valores de viscosidad intermedios, es interesante mencionar que se presentaron diferencias estadísticas significativas $(P>0,05)$ en cada uno de los valores reportados. Este comportamiento puede indicar que no existe una interacción entre la GG y la CPS aunque más estudios son necesarios para confirmar esta hipótesis.

Es interesante destacar que todas las biopelículas mostraron menores valores de compliancia retardada en comparación a los valores de compliancia instantánea $\left(\mathrm{J}_{1}<\mathrm{J}_{0}\right)$, esto es debido a que las biopelículas necesitan deformarse inicialmente antes que el flujo se estabilice, y deformarse nuevamente por la aplicación del esfuerzo, este comportamiento también fue observado por González et al. (2012) en geles elaborados con mezclas de gelana de bajo y alto acilo. Los valores de $\mathrm{J}_{0}$ son inversamente proporcionales a los valores de viscosidad, considerando que la compliancia es el opuesto del esfuerzo aplicado (Jiménez et al. 2005). Los valores de $J_{0}$ son cercanos a cero, esto indica que el área exponencial es pequeña mostrando un comportamiento con una tendencia similar a un sólido elástico ideal, por otro lado los valores de viscosidad alcanzan valores mayores que $40 \times 10^{6}$ veces el valor de la viscosidad del agua a $20^{\circ} \mathrm{C}$. Los porcentajes de incrementos de $J_{0}$ con respecto a $J_{1}$ para las biopelículas de $G G$, CPS y sus mezclas 25GG/75CPS, 50GG/50CPS y 75GG/25CPS fueron 715, 1470, 633, 431, 1050 respectivamente, lo cual muestra que los valores disminuyen con el aumento de GG.El $\lambda_{\text {ret }}$ mostró una amplia variación de $(3,15 \times$ $10^{1}$ a $\left.4,12 \times 10^{-2} \mathrm{~s}\right)$. Es importante destacar que $\lambda_{\text {ret }}$ es el tiempo necesario para que la deformación disminuya hasta (e-1) de su valor final. El $\lambda_{\text {ret }}$ aumentó a medida que aumentaba la proporción de CPS en la biopelícula, esto puede ser un indicativo de la pérdida de flexibilidad de la biopelícula.

Tabla 1 parámetros obtenidos del ajuste de las curvas de Creep para el modelo de Burgers

\begin{tabular}{|c|c|c|c|c|c|c|c|}
\hline \multicolumn{2}{|c|}{ Mezclas (\%) } & \multirow{2}{*}{$\eta(\mathrm{Pas})$} & \multirow{2}{*}{$\mathrm{J}_{0}\left(\mathrm{~Pa}^{-1}\right)$} & $J_{1}(\mathrm{~Pa}-1)$ & $\lambda_{\text {ret }}(\mathrm{s})$ & $1 / J_{0}$ & \multirow{2}{*}{$\%$ Recuperación } \\
\cline { 1 - 5 } CPS & GG & & & & & 63 \\
\hline 0 & 100 & $8,23 \times 10^{4}$ & $7,15 \times 10^{-4}$ & $1,00 \times 10^{-6}$ & $4,12 \times 10^{-2}$ & $1,39 \times 10^{3}$ & 65 \\
\hline 25 & 75 & $5,86 \times 10^{5}$ & $6,33 \times 10^{-4}$ & $1,00 \times 10^{-6}$ & $1,13 \times 10^{-2}$ & $1,57 \times 10^{3}$ & 71 \\
\hline 50 & 50 & $6,77 \times 10^{5}$ & $4,31 \times 10^{-4}$ & $1,00 \times 10^{-6}$ & $1,22 \times 10^{1}$ & $2,32 \times 10^{3}$ & 71 \\
\hline 75 & 25 & $7,45 \times 10^{5}$ & $1,05 \times 10^{-3}$ & $1,00 \times 10^{-6}$ & $2,18 \times 10^{1}$ & $9,52 \times 10^{2}$ & 74 \\
\hline 100 & 0 & $4,67 \times 10^{6}$ & $1,47 \times 10^{-3}$ & $1,00 \times 10^{-6}$ & $3,15 \times 10^{1}$ & $6,80 \times 10^{2}$ & \\
\hline
\end{tabular}

Finalmente se puede decir que aunque el aumento en las concentraciones de CPS en la elaboración de biopelículas conlleva a un incremento en el comportamiento elástico de las biopelículas, es necesario incorporar mayor cantidad plastificante, debido a que el $\lambda_{\text {ret }}$ evidenció una posible pérdida de flexibilidad (Galieta et al. 2005; Mchugh et al. 1994), lo cual se puede suplir incrementando la concentración de plastificante.

\section{CONCLUSIONES}

Todas las biopelículas estudiadas presentaron un comportamiento reológico viscoelástico, en donde la componente elástica fue mayor que la componente viscosa (G'>G'), lo que indica que son sistemas con características predominantemente elásticas, este comportamiento fue directamente proporcional a las concentraciones de CPS utilizadas. Sin embargo, las biopelículas con mayor concentración de CPS tienden a perder su flexibilidad, por lo cual es recomendable aumentar la concentración de plastificante. El comportamiento de los módulos dinámicos puede ser un indicativo de la formación de redes interdependientes de la GG y el CPS. Por tanto, es posible utilizar estos materiales (GG y CPS) individualmente $\mathrm{o}$ en mezclas, para la obtención de biopelículas activas y recubrir diversos sistemas alimentarios sólidos. Igualmente es recomendable llevar a cabo estudios de factibilidad de este tipo de biopelículas y de estimación de vida útil de los productos alimenticios que sean sometidos a recubrimiento. La caracterización reológica de las biopelículas es un factor importante en el diseño de biopelículas activas y su correcta aplicación en los diversos sistemas alimentarios. 


\section{REFERENCIAS}

Acevedo, D. y otros dos autores, Caracterización reológica de pastas untuosas artesanal y tecnificada de ajonjolí (Sesamun indicum) cultivado en Zambrano-Bolivar (Colombia), Inf Tecnol, 25(4), 73-78 (2014a)

Acevedo, D, y otros dos autores, Caracterización reológica del suero costeño de Turbaco, Arjona, El Carmen de Bolivar y uno comercial (Colombia), Inf Tecnol, 25(3), 3-10 (2014b)

Clark, A, y S. Ross-Murphy, Structural and mechanical properties of biopolymers gels, In Dusek, K (Eds) Advances in Polymer Science. Vol 83 Springer Verlag, Germany, 57-192 (1987)

Chillo, S, y otros cinco autores, influence of glicerol and chitosan on tapioca starch-based edible film properties, Journal of Food Engineering, 88:159-168 (2008)

Chen, $\mathrm{C}$, y otros dos autores, Rheological and physical characterization of film-forming solutions and edible films from tapioca starch/decolorized hsian-tsao leaf gum, Food Hidrocol, 23(8), 2132-2140 (2009)

Deiber, J, y otros dos autores, Rheological characterization of edible films made from collagen colloidal particle suspensions, Food Hidrocol, 25(5), 1382-1392 (2011)

Franssen, L, y J. Krochta, Edible coating containing natural antimicrobials for processed foods, in Soller, $\mathrm{S}$ (eds) Natural Antimicrobials for the Minimal Processing of Foods, CRC Press, Boca Raton, FL., USA (2003)

Galieta, G, y otros tres autores, aumento de la vida util postcosecha de tomate usando una pelicula de proteina de suero de leche, Rev Iber tecnol post, 6(2): 117 - 123 (2005)

González, A, y C. Alvarez, Soy protein e Poly (lactic acid) bilayer films as biodegradable material for active food packaging, Food Hydrocol, 33: 289-296 (2013)

González, R. Efecto de la concentración de gelana de alto y bajo acilo en la microencapsulación del cultivo iniciador Lactococcus lactis en la obtención de quesos tipo Manchego, Tesis de Doctorado, Departamento de Biotecnología y Bioingeniería, CINVESTAV, México D.F., México, (2011)

González, R, y otros tres autores, Rheological characterization and activation energy values of binary mixtures of gellan, Eur Food Res Technol, 235:305-313 (2012)

González, R y otros dos autores, Efecto de la microencapsulación sobre las propiedades reológicas y fisicoquimicas del yogurt blando, Inf. Tecnol. 25(6), en prensa (2014)

Hua $\mathrm{Li}, \mathrm{J}$ y otros cuatro autores, Preparation and characterization of active gelatin-based films incorporated with natural antioxidants, Food Hydrocol, 37: 166-173 (2014)

Jimenez, $\mathrm{H}$, y otros dos autores, Viscoelastic characterization of gum arabic and maize using the maxwell model, Carb Pol, 62: 11-18 (2005)

Lau, P, y otros tres autores, Absolute Quantitation of Bacterial Biofilm Adhesion and Viscoelasticity by Microbead Force Spectroscopy, Biophy J, 96(7), 2935-2948 (2009)

León, $\mathrm{P}, \mathrm{y}$ otros cinco autores, Rheological characterization of deacylated/acylated gellan films carrying I-(+)ascorbic acid, Food Hydrol, 23(7), 1660-1669 (2009)

Liang, $\mathrm{J}, \mathrm{y}$ otros cinco autores, Influence of glicerol on the molecular mobility, oxygen permeability and microstructure of amorphous zein film, Food Hydrocol, en prensa (2014)

Lin, D, y Y, Zhao, Innovations in the developmet and application of edible coating for fresh and minimally processes fruit and vegetables. Comp Rev food sci food saf, 6(1), 60 - 74 (2007)

Lucey, J. y otros tres autores, Microstructure, permeability and appearance of acid gels made from skim milk, Food Hydrocol, 12(2), 159-165 (1998)

Malkin, A, Rheology fundamentals. Ed. Chem Tec Publishing. Pp: 1 - 13 (1994)

Mchugh, $T$ y otros dos autores, plasticized whey protein edible film: water vapor permeability properties. $J$ Food Sci, 59(2): 416 - 419 (1994)

Peressini, D, y otros cuatro autores, Starch-methylcellulose based edible films: rheological properties of filmforming dispersions, J Food Eng, 59:25-32 (2003)

Silva, A, y otros cuatro autores, structural properties of films and rheology of film-forming solutions based on chitosan and chitosan-starch blend enriched with murta leaf extract, Food hydrocol,31:458-466 (2013)

Steffe, J, Rheological methods in food process engineering. Ed. Freeman Press. pp 1-91 (1996) 